\title{
Large errors, but no depth compression, in walked indications of exocentric extent
}

\author{
JOHN W. PHILBECK, SHANNON O'LEARY, and AUDRA LYN BLOHM LEW \\ George Washington University, Washington, D.C.
}

\begin{abstract}
Observers can sight a target $20 \mathrm{~m}$ away or more and then walk to it accurately without vision. In contrast to this good performance, this article shows that walked indications of the exocentric separation of two locations exceed the required values by over $70 \%$ when vision is obscured. Significantly, these large errors are coupled with a robust lack of depth foreshortening, even under conditions in which visual matches and verbal estimates of extent exhibit strong evidence of depth compression. This article presents evidence that the overshooting errors are due largely to recalibration of locomotor control produced by prolonged exposure to nonvisual walking. The robust lack of depth foreshortening, meanwhile, could reflect a corresponding isotropy in the spatial representation controlling the walking response. More research is needed to confirm this interpretation, however.
\end{abstract}

Over 20 years of research have shown that people are extraordinarily good at walking to locations in the nearby environment. In egocentric walking tasks (which involve a direct walk to a single target), the average observer can view a target $20 \mathrm{~m}$ away or more and then walk to it quite accurately, even without vision (e.g., Loomis, Da Silva, Fujita, \& Fukusima, 1992; Rieser, Ashmead, Talor, \& Youngquist, 1990; Thomson, 1983). This accuracy is striking because other observations suggest that there are large distortions in perceived spatial layout at such distances. Traffic signs painted onto the street provide an everyday example. Although greatly elongated along one axis, the signs often appear to be much less elongated when viewed from the perspective of a pedestrian. A common interpretation of such distortions of perceived shape is that depth intervals are perceptually compressed. Even at viewing distances as little as $3 \mathrm{~m}$, depth intervals are perceptually compressed by $15 \%$ or more (Philbeck, 2000), and this compression increases to more than $50 \%$ at distances of 10-20 m (Beusmans, 1998; Loomis et al., 1992; Norman, Todd, Perotti, \& Tittle, 1996; Toye, 1986; Wagner, 1985).

Despite this strong evidence of perceptual distortion, nonvisual walking responses in egocentric tasks are accurate under similar viewing conditions, and even walked

Portions of this research were supported by ONR Grant N00014-951-0573 to Jack Loomis. A preliminary report of portions of this work was presented in May 2000 at the annual meeting of the Association for Research in Vision and Ophthalmology in Ft. Lauderdale, FL. The authors thank Jack Loomis, Marlene Behrmann, Hal Sedgwick, and an anonymous reviewer for helpful comments on earlier versions of this manuscript. Correspondence concerning this article should be addressed to J. W. Philbeck, Department of Psychology, George Washington University, 2125 G. Street, N.W., Washington, DC 20052 (e-mail: philbeck@gwu.edu).

Note-This article was accepted by the previous editorial team, headed by Neil Macmillan. indications of exocentric intervals show no hint of foreshortening. Exocentric tasks require observers to assess the relation between two points in space that are displaced from the observer. Loomis et al. (1992) asked observers to view two markers on the ground, delineating either a depth interval or a frontal interval (parallel to an imaginary line passing through the two eyes). Observers then closed their eyes and attempted to walk without vision to each endpoint of the interval in sequence. Remarkably, when responses to depth intervals were compared with responses to physically equal frontal intervals, there was virtually no evidence of depth compression. In contrast, when observers attempted to match the separation of two depth markers and two frontal markers, the resulting configurations were strongly distorted, very much in keeping with the distorted appearance of street signs. The lack of depth compression demonstrated in the exocentric walking task contrasts sharply with visual matches of exocentric intervals but appears to accord well with the good accuracy typical in egocentric walking tasks.

Lack of compression in depth-walking responses, relative to frontal-walking responses, however, does not necessarily imply that the walked intervals are accurate. Although Loomis et al. (1992) were primarily interested in the lack of depth compression, their observers in fact overshot the required intervals by up to $50 \%$. There was no depth compression, because the same amount of overshooting was present for both depth and frontal intervals. The lack of depth compression seems to suggest that the observers were, at some level, sensitive to the true (undistorted) spatial layout, but this explanation is not entirely satisfying in light of the large, systematic overwalking errors. If the layout is accurately registered and this information is responsible for the lack of depth foreshortening in walking responses, why does this information not also result in accurately scaled walking? These findings still await a satisfactory explanation. 
The goal of the present experiments was to investigate the source of the large overwalking errors and the lack of depth foreshortening exhibited in exocentric walking tasks. In Experiment 1, we tested the hypothesis that the difference between the walking and the visual-matching indications of exocentric intervals in Loomis et al. (1992) was due to differences in the stimulus configurations used for the two tasks. In this view, both visual matches and nonvisual walking responses were controlled by a common underlying representation (or by separate representations containing identical information), but the stimulus configuration used in the walking task caused this representation to be less foreshortened in depth. This hypothesis predicts that using the same stimulus configuration for motoric and nonmotoric responses should yield a similar pattern of responding for both tasks. In Experiment 1 , we tested this prediction.

An alternative possibility stems from the fact that the visual-matching responses in Loomis et al. (1992) were conducted at the observation location, whereas the walked indications of the stimulus interval were conducted at the interval itself, several meters from the observation point. As we will discuss below, there are several reasons why this difference may have contributed to the lack of depth foreshortening exhibited in the walking responses. In Experiment 2 , we tested this possibility by asking observers to indicate the magnitude of the stimulus extent by walking a matching distance from the observation location.

In Experiment 3, we further explored the robustness of the lack of depth foreshortening in nonvisual walking by increasing the range of stimulus sizes and by varying the direction in which participants walked to indicate the stimulus extents. Finally, in Experiment 4, we tested the possibility that the large overwalking errors could have been the result of recalibration of locomotor control as a result of prolonged exposure to nonvisual walking.

\section{EXPERIMENT 1}

Loomis et al. (1992) presented observers with four markers on each visual-matching trial (two markers each to specify a depth and a frontal interval), but only two markers on each walking trial. This dissimilarity in the stimulus configuration is potentially significant, because the presence of additional flanking elements can have an impact on how attention is allocated while the configuration is being viewed. In particular, in the walking task, the observers did not have to directly compare depth and frontal components of the stimulus configuration and, therefore, could focus their attention on a smaller number of markers; by contrast, in the matching task, the observers had to distribute their attention among a larger number of stimulus elements and explicitly compare depth and frontal intervals. This is a critical difference, because tasks that encourage focusing of attention on individual elements, as opposed to distributing attention across a configuration, are associated with reduced evidence of perceptual distortions (Mack, Heuer, Villardi,
\& Chambers, 1985; Rock, 1986). Similar ideas have been expressed more recently (Brenner \& Smeets, 1996; Franz, Fahle, Bülthoff, \& Gegenfurtner, 2001; Vishton, Rea, Cutting, \& Nuñez, 1999). Loomis et al.'s (1992) two-marker walking task may encourage exactly this kind of attentional focusing, resulting in perceptual representations that are less depth-foreshortened than the representations created when observers view four markers in the matching task. Another possible consequence of there being fewer stimulus elements is that observers may be more likely to consciously compensate for distortions in perceived depth. Before evaluating other possible sources of the discrepancy in responses shown for the two tasks, therefore, it is crucial to verify that the discrepancies still exist when the stimulus context does not vary across response type. The goal of Experiment 1 was to compare exocentric walking responses with one type of nonmotoric response (verbal reports) under conditions in which the number of stimulus elements was held constant.

On every trial in Experiment 1, observers saw a single rod lying on the ground, oriented either in depth or frontally. On some trials, the observers gave verbal estimates of the rod length, and these estimates were subsequently compared across trials to assess the degree of depth foreshortening. On other trials, following Loomis et al. (1992), the participants closed their eyes after viewing the rod and attempted to walk out to each endpoint in sequence. The observers viewed the stimuli either monocularly or binocularly; monocular viewing removes the powerful depth cue of binocular disparity and might, therefore, magnify any evidence of depth foreshortening in the responses. Given the inaccuracies in exocentric tasks that had been observed previously in both motoric and nonmotoric responses, we were interested in assessing the participants' best estimates of layout when all the available information was considered. Accordingly, the instructions for both verbal and walking tasks in Experiment 1 emphasized responding on the basis of objective accuracy (Carlson, 1977).

\section{Method}

Participants. Sixteen adults ( 8 males and 8 females) participated in this experiment for pay. Their ages ranged from 20 to 33 years (median $=22$ ). All were naive as to the purpose of the experiment.

Design. Equal numbers of males and females were assigned to the monocular and the binocular groups. The response modality (verbal or walking) was blocked and counterbalanced. On each trial, a single rod was presented at one of three distances $(4,7$, or $10 \mathrm{~m}$ ) and one of two orientations (depth or frontal). A $137 \times$ $2.54 \mathrm{~cm}$ rod was presented on two thirds of the trials; these experimental trials were the only ones analyzed. Two measurements per condition were collected, for a total of 24 trials including both response modalities. The remaining 12 trials (randomly interspersed) used four other rod lengths: 91, 114, 160, and $206 \mathrm{~cm}$. This provided some variation in length across trials, to minimize response biases that might accompany repeated presentations of the same stimulus. The nonexperimental rods were randomly paired with each combination of distance and orientation; this meant that each individual participant generally did not see exactly the same set of 
stimuli in the depth and the frontal orientations. This was done to minimize the possibility that the observers would be biased toward creating isotropic responses (i.e., exhibiting neither depth foreshortening nor depth expansion) because they assumed that the same stimuli always appeared at both orientations.

Stimuli and Apparatus. The experiment took place outdoors in a flat grassy field, $12 \times 56 \mathrm{~m}$. To minimize auditory information, the participants wore tight-fitting hearing protectors (noise reduction rating: $-20 \mathrm{~dB}$ ). The monocular group wore an eye patch over the nondominant eye while viewing the stimuli; all the observers used a blindfold to obscure vision before walking.

Procedure. Prior to testing, the participants were familiarized with nonvisual walking by walking without vision for approximately 3 min under the guidance of a sighted experimenter. The participants viewed each rod for $5 \mathrm{sec}$, then lowered the blindfold and initiated their response. On verbal trials, the participants gave a verbal estimate of the rod's length under the following objective instructions: "Remember that you are looking at the stick from an angle, and this can make things appear to be a different size than they are physically. What I want you to tell me about, however, is the physical size, as if you were standing over the stick with a tape measure and reading off the number." On walking trials, they attempted to walk out to the rod's location, stopping briefly at each endpoint, again under objective instructions. The participants walked at their own self-determined speed (generally about $1.0-1.5 \mathrm{~m} / \mathrm{sec}$ ). The straight-line distance from the origin to the first stopping point was recorded, as well as the distance between the two stopping points. No error feedback was given, and analyses of variance (ANOVAs) were used to analyze the data. The data were averaged across repetition for each observer before analysis.

\section{Results and Discussion}

Experiment 1 compared verbal reports and walked indications of rod length under conditions in which the num- ber of stimulus elements was held constant. The results clearly showed that large differences remained between these two response types even when the stimulus context was controlled (see Figure 1A). Figure 2A plots the data of all four experiments as response ratios (depth/frontal), to emphasize the amount of depth foreshortening; Figure $2 \mathrm{~B}$ plots the data in terms of the mean responses, averaged over depth and frontal stimuli, to emphasize the amount of overwalking. The verbal and walking responses distinctly differed in terms of their sensitivity to the orientation of the stimulus intervals [response type $\times$ orientation: $F(1,14)=4.64, p<.05$ ], in keeping with Loomis et al. (1992). Planned comparisons showed that the walking responses exhibited virtually no depth compression (orientation: $p=.15$; Figure 1A; see also Figure 2A), whereas the verbal length estimates did show a sizable amount of foreshortening (orientation: $p<.001$ ). In a significant departure from Loomis et al.'s (1992) data, however, this foreshortening was less than half that found in the visual matches of the earlier work $(18 \%$ here vs. Loomis et al.'s 40\%). Thus, the differences in the number of stimulus elements across tasks in Loomis et al. (1992) may well account for some of the discrepancy between the walking and the visual-matching tasks, but the fact that there is still evidence of depth foreshortening in the verbal reports obtained here suggests that this does not entirely account for the discrepancy.

Although there was no evidence of depth foreshortening in the walking responses, the responses themselves were highly inaccurate (Figures 1A and 2B). On average,
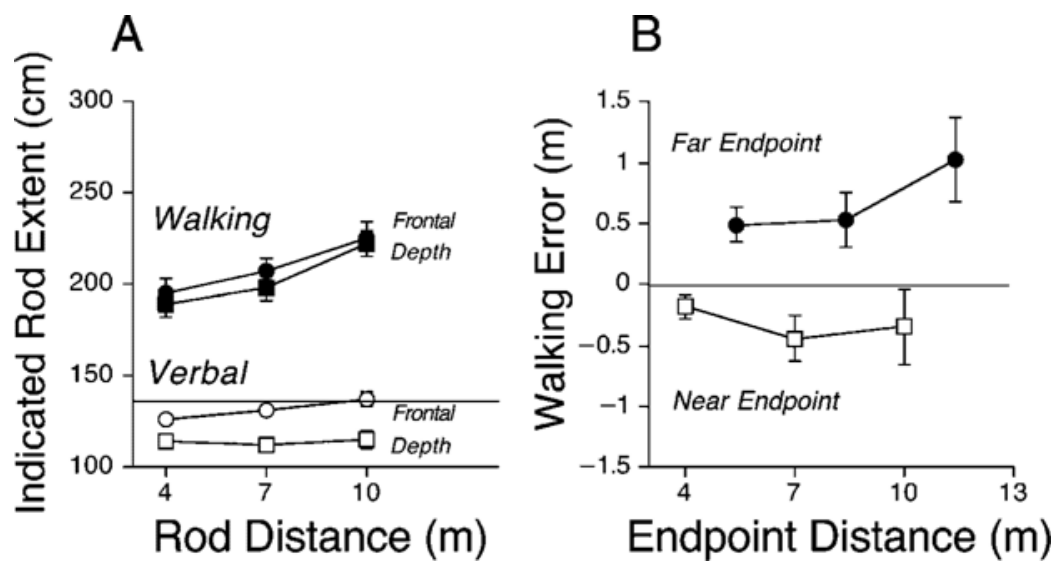

Figure 1. (A) Mean indicated rod extent for frontal and depth rod orientations. Walked indications were conducted at the rod's location. The horizontal line indicates accurate performance. Although walked indications of frontal and depth rods are quite similar, there were large overshooting errors. Verbal responses were more accurate but showed evidence of foreshortening. All error bars denote plus or minus one standard error of the mean. For clarity, the data are averaged over monocular/binocular viewing; responses under monocular viewing were about $12 \%$ smaller than those under binocular viewing but did not interact with any other variable. (B) Mean signed error in walking from the origin to the near and far endpoints of a $137-\mathrm{cm}$ rod oriented in depth. The endpoints served as sequential targets in the same trial. The horizontal line indicates accurate performance. There is a clear difference in the pattern of errors, depending on the target endpoint of the rod. All error bars denote plus or minus one standard error of the mean, and the data are averaged over monocular/binocular viewing conditions. 

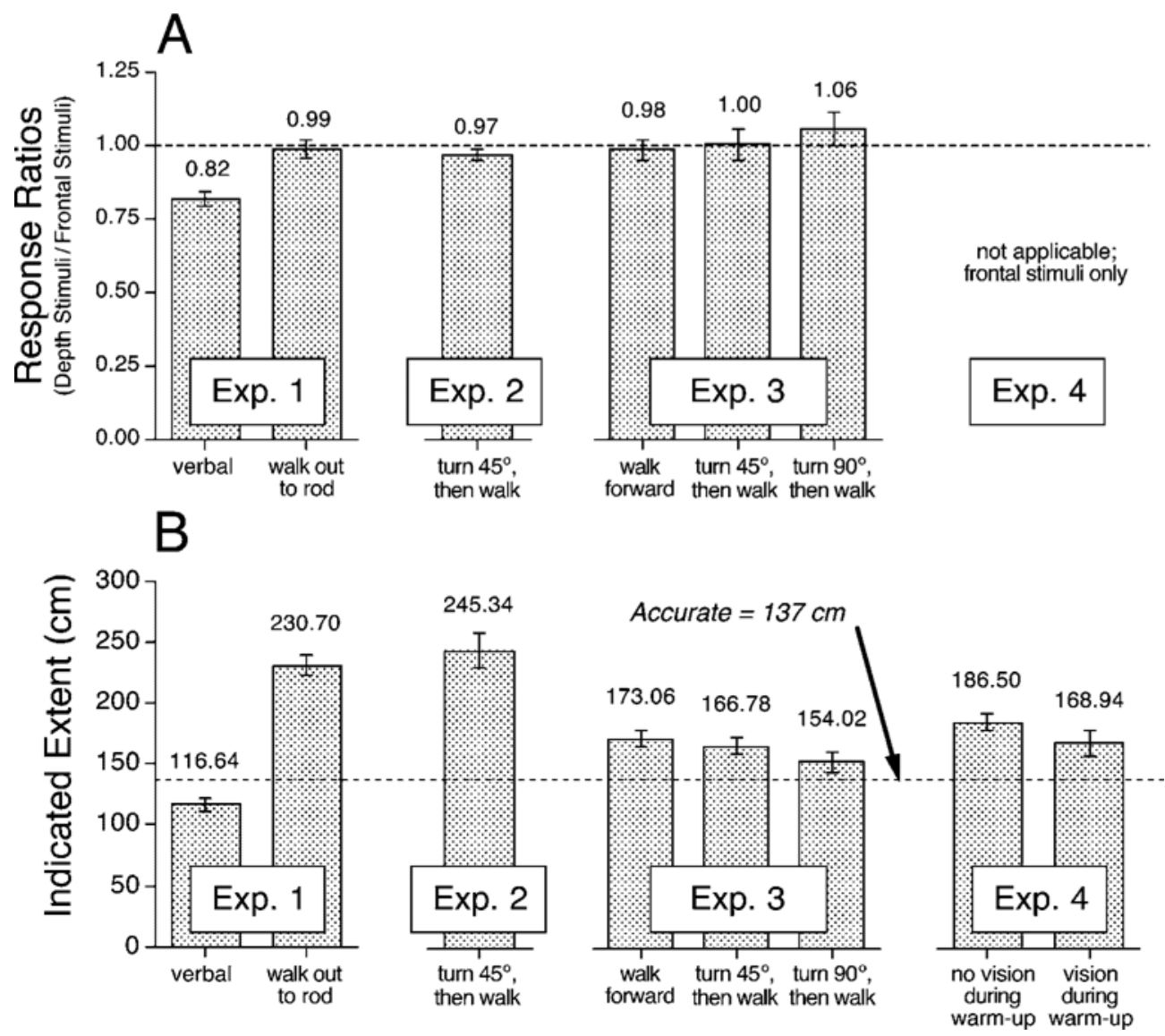

Figure 2. Summary figure showing the results of various response conditions from Experiments 1-4. The data for Experiments 1 and 2 are averaged over viewing distance and monocular/binocular viewing; Experiment 2 is additionally collapsed over the task cue manipulation. For purposes of comparison, the figures reflect responses to the $137-\mathrm{cm}$ stimulus extent only. All error bars denote plus or minus one standard error of the mean, calculated between observers after collapsing over the other variables. (A) Mean response ratios (depth stimuli/frontal stimuli). The dashed horizontal line indicates isotropic responding. Response ratios were not statistically analyzed, but they are related to the effect of stimulus orientation in the signed response error analysis; values near 1 indicate isotropic responding, whereas values deviating from 1 indicate an increasing dependence of responses on stimulus orientation. Note that response ratios could not be calculated for Experiment 4 because no depth stimuli were presented. (B) Mean indicated extents. The dashed horizontal line indicates accurate responding.

the walking responses overshot the correct rod length by about $68 \%$ - even more than the overwalking in Loomis et al. (1992). Some participants, in fact, walked over $350 \mathrm{~cm}$ when attempting to mark out the length of a 137-cm rod (see Figure 3, Experiment 1). These overshooting errors, then, were large and highly reliable. In striking contrast, the verbal estimates were much more appropriately scaled, albeit with some foreshortening.

The large main effect of response type $[F(1,14)=$ $130.66, p<.001]$ was accompanied by main effects of orientation and viewing distance $[F(1,14)=11.01$ and $F(2,28)=39.75$, respectively; both $p$ s $<.01]$. The two response types were differently affected by the rod viewing distance [response type $\times$ distance: $F(2,28)=16.56$, $p<.001]$. Planned comparisons showed that the verbal estimates of rod length were not influenced by viewing dis- tance (all pairwise $p \mathrm{~s}>.05$ ), whereas walked indications of rod length tended to increase as the participants had further to travel to reach the first endpoint (all pairwise $p \mathrm{~s}<.01)$. Monocular group responses were about $12 \%$ smaller than those of the binocular group $[F(1,14)=8.57$, $p<.05]$, but this small effect did not interact with any other variable. This may reflect chance between-groups differences.

We also assessed the observers' accuracy in arriving at the location of each individual rod endpoint. The most direct way of addressing this is to focus on trials involving depth rods only, since these trials do not require large body rotations that might engage different spatial-updating processes (Berthoz et al., 1999). An ANOVA on the signed walking errors (depth rods only) showed a main effect of endpoint $[F(1,14)=148.49, p<.001]$ and an 

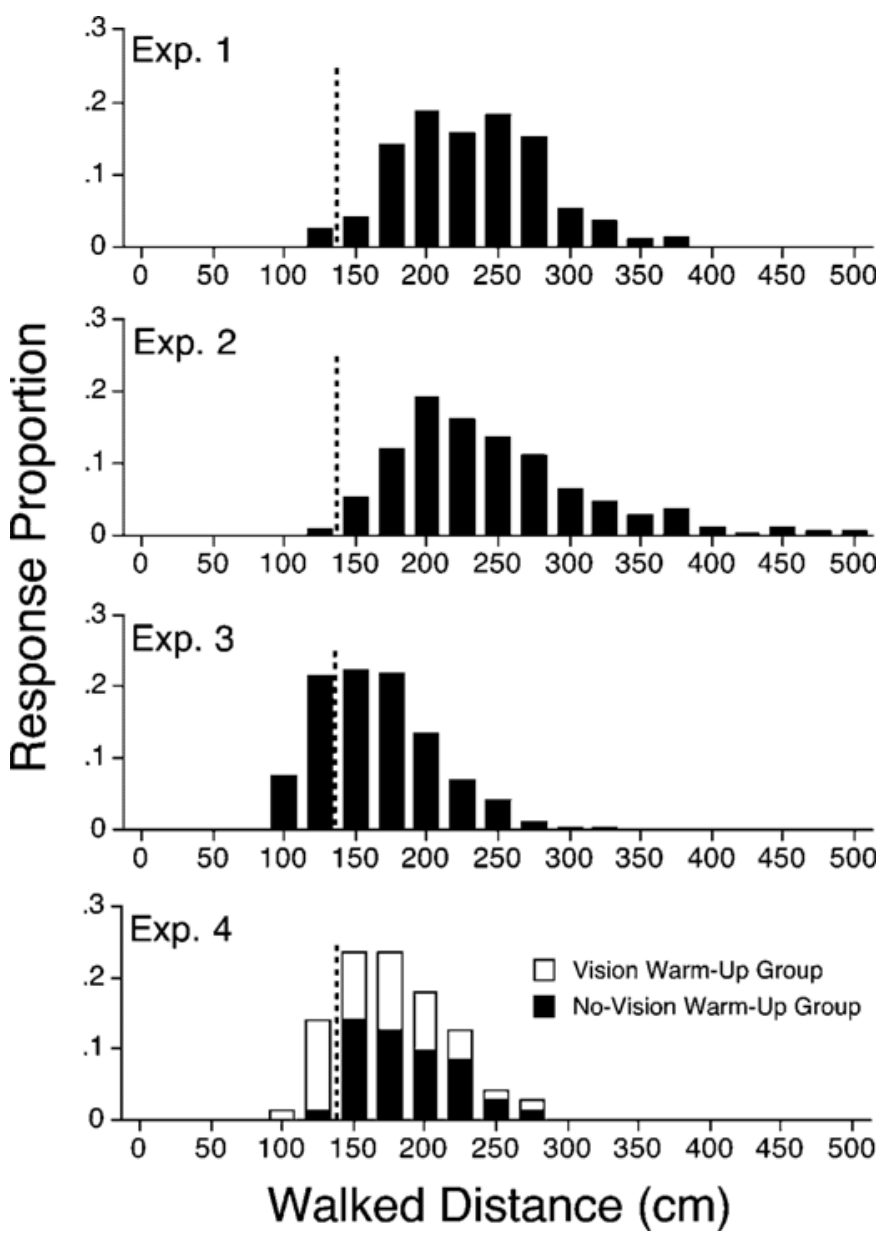

Figure 3. Frequency histograms for Experiments 1-4, showing the relative proportion of various walked distances that observers produced when attempting to reproduce the stimulus extent. The data shown are for the 137- $\mathrm{cm}$ stimulus only. The bin width is $25 \mathrm{~cm}$. Bars to the left of the dashed vertical lines represent responses that undershot the required value (Experiment 1, 5 out of 192 responses; Experiment 2, 3 out of 384 responses; Experiment 3, 66 out of 252 responses; Experiment 4, no-vision warm-up group, 1 out of 36 responses; Experiment 4, vision warm-up group, 10 out of 36 responses).

endpoint $\times$ distance interaction $[F(2,28)=17.44, p<$ $.001]$. There were some variations in error across certain combinations of target distance and endpoint, but planned comparisons indicated that there were reliable endpoint effects at all the target distances (all $p$ s $<.001$ ). Figure $1 \mathrm{~B}$ shows that the near endpoint was slightly undershot, whereas the far endpoint was slightly overshot. Thus, there was no clear evidence in Experiment 1 that the participants accurately arrived at one endpoint and then made errors in arriving at the other. Nevertheless, as the endpoint main effect and the vertical separation of the two curves in Figure 1B show, the participants clearly did not produce the same kind of errors for both endpoints. Instead, there was a context effect in which the pattern of errors at each rod distance was strongly influ- enced by which endpoint was the target. Finally, in Loomis et al. (1992), observers tended to walk about $50 \mathrm{~cm}$ too far no matter how far apart the endpoints were physically separated; Experiment 1 was not designed to investigate this issue systematically, but there were no striking differences in overwalking across rod lengths (see Table 1).

\section{EXPERIMENT 2}

Experiment 1 showed that when participants use verbal reports and nonvisual walking to indicate exocentric spatial intervals, their responses exhibit distinctly different patterns even for equivalent stimulus configurations. Verbal responses yield evidence of depth compression, whereas walking responses do not. Despite the similar- 
Table 1

Mean Signed Walking Error (and Standard Deviations) for Seven Stimulus Extents

\begin{tabular}{|c|c|c|c|c|c|c|c|c|c|c|}
\hline \multirow[b]{3}{*}{ Stimulus } & \multicolumn{10}{|c|}{ Experiment } \\
\hline & \multicolumn{2}{|c|}{$1 *$} & \multicolumn{2}{|c|}{$2 *$} & \multicolumn{2}{|c|}{$3 \dagger$} & \multicolumn{2}{|c|}{4 (no vis) $t$} & \multicolumn{2}{|c|}{4 (vis) $\ddagger$} \\
\hline & $M$ & $S D$ & $M$ & $S D$ & $M$ & $S D$ & $M$ & $S D$ & $M$ & $S D$ \\
\hline 91 & 86 & 52 & 87 & 46 & - & - & - & - & - & - \\
\hline 114 & 102 & 51 & 120 & 80 & - & - & - & - & - & - \\
\hline 137 & 94 & 34 & 108 & 58 & 27 & 23 & 49 & 24 & 32 & 37 \\
\hline 160 & 80 & 42 & 112 & 59 & - & - & - & - & - & - \\
\hline 206 & 73 & 40 & 109 & 64 & - & - & - & - & - & - \\
\hline 244 & - & - & - & - & 25 & 49 & 76 & 40 & 44 & 46 \\
\hline 351 & - & - & - & - & 18 & 70 & 154 & 85 & 49 & 71 \\
\hline
\end{tabular}

Note-All values are expressed in centimeters. *Averaging over viewing distance, stimulus orientation, and monocular/binocular viewing. The $137-\mathrm{cm}$ stimulus was the only experimental extent; the others were not fully crossed with viewing distance and orientation. †Averaging over response direction (forward, turn $45^{\circ}$, turn $90^{\circ}$ ) and stimulus orientation. $\ddagger$ Results from Test 1 ; no vis, data from group that walked 3 min without vision prior to testing; vis, data from group that walked 3 min with vision prior to testing.

ity of the stimulus configurations, the two tasks still differ along other dimensions. The most salient difference involves the fact that the walking task entails locomoting out to the stimulus location and stopping at two endpoints in succession, whereas the verbal task and the visualmatching task in Loomis et al. (1992) do not. This methodological distinction can be characterized as creating task differences in terms of egocentric versus exocentric distance perception (e.g., Loomis et al., 1992; Loomis, Da Silva, Philbeck, \& Fukusima, 1996), relative versus absolute distance perception (e.g., Franz et al., 2001; Vishton et al., 1999), and definite versus relative distance perception (e.g., Bingham, 1993; Bingham \& Pagano, 1998). Similar ideas have been expressed by a variety of other authors (Abrams \& Landgraf, 1990; Brenner \& Smeets, 1996; Gillam \& Chambers, 1985; Mack et al., 1985; Marteniuk \& Roy, 1972; Soechting \& Flanders, 1992). In general, these ideas suggest that the way observers process a particular stimulus configuration depends critically on what aspect of the stimuli is emphasized. Because the walking task entails locomoting out to the stimulus location, it may lead to an emphasis on the endpoint locations more than the verbal and the visualmatching tasks do, and this emphasis may in turn enhance the spatial representation used to control walking. To make the walking task more similar to the verbal and matching tasks, in Experiment 2, we asked participants to pace off the rod length at the observation location, instead of at the rod's location. This manipulation made the walking task much more closely matched to the verbal task of Experiment 1. This may well bring the walking responses into closer register with the verbal judgments and visual matches of extent.

An influential framework espoused by Milner, Goodale, and others (e.g., Bridgeman, 1999; Haffenden \& Goodale, 1998; Marotta, Behrmann, \& Goodale, 1997; Milner \& Goodale, 1995; Pagano \& Bingham, 1998; Proffitt, Creem, \& Zosh, 2001; Wraga, Creem, \& Proffitt, 2000) provides another possible reason to suspect that length walking at the observation location will exhibit the depth foreshortening more typical of visual matches of length. These authors have suggested that there is a neuroanatomical stream of visual processing that constructs relatively accurate spatial representations for the purpose of on-line control of actions. There is evidence, however, that pantomiming an action at a location displaced from its target engages a different processing stream - one thought to be linked with conscious perceptual representations (Goodale, Jakobson, \& Keillor, 1994). Indicating the rod's length near the observation point, then, may be especially likely to reveal perceptual depth distortions.

The typical finding when people walk without vision to indicate the location of a single previously viewed target is that walking is generally quite accurate out to $22 \mathrm{~m}$ or more (Rieser et al., 1990). This means that constant (signed) errors do not depend strongly on target distance within this range. The results of Experiment 1 showed that when people walk without vision to indicate the locations of two rod endpoints, their performance differs from that exhibited in typical single-target tasks. Specifically, there is a different pattern of errors for each rod endpoint in the two-endpoint task (see Figure 1B) - that is, the constant errors now vary systematically with target distance. This endpoint effect could also be related to the requirement of walking to two locations in sequence. For example, this task might tend to generate errors of anticipation and perseveration for the near and the far endpoints, respectively. To investigate these possibilities, Experiment 2 included egocentric walking trials in which a single rod endpoint was specified and observers were asked to walk without vision to just that one location. This removed the requirement of indicating more than one location within a trial and might, therefore, be expected to produce results more in keeping with the standard single-target nonvisual walking task.

The inclusion of these egocentric trials also permitted a test of the idea expressed earlier, that the way observers process a particular stimulus configuration depends on what stimulus feature is emphasized by the task. Intro- 
ducing uncertainty as to what aspect of the stimulus should be indicated (rod length vs. endpoint location) could expose this influence by encouraging observers to rely on a strategy that will support both judgments. To achieve this uncertainty, the response type was specified after vision was obscured for one group of participants. In a second group, the response type was specified before vision was obscured, thereby allowing this group to use vision to prepare for a specific response. One might expect walked indications of rod length to show somewhat less depth foreshortening when the response type is specified after vision is obscured; the possible requirement of walking out to the rod should encourage observers to pay more attention to the rod endpoint locations. As we have seen, several frameworks would predict that this emphasis on the endpoint locations should enhance the accuracy of the spatial representation that presumably underlies the response. Finally, to investigate the possible effect of removing binocular cues suggested by Experiment 1 , all the observers participated in both monocular and binocular trials.

\section{Method}

Participants. Sixteen adults ( 8 males and 8 females) were paid for their participation. Their ages ranged from 18 to 27 years (median $=21$ ). All were naive as to the purpose of the experiment.

Design. The observers walked without vision to a specified endpoint of the rod (endpoint-walking trials) or walked an amount equal to the rod length, starting from the observation location (length-walking trials). To manipulate response certainty, the type of response was specified before vision was obscured for 8 participants and afterward for the rest. Each observer participated in a monocular and a binocular session, in a counterbalanced block order and with about a week separating the blocks. On each trial, a single rod was presented at one of three distances $(4,7$, or $10 \mathrm{~m})$ and one of two orientations (depth or frontal). The $137-\mathrm{cm}$ rod was presented on approximately two thirds of the trials, with the remaining one third (randomly intermixed) being drawn from the four other rod lengths: 91, 114, 160, and $206 \mathrm{~cm}$. As in Experiment 1, this was done to avoid presenting exactly the same set of stimuli at both orientations, a situation that might bias the observers toward isotropic responding. The $114-\mathrm{cm}$ rod was not presented to the group that was notified of the task before vision was obscured. This shortened the running time by several trials and slightly changed the distribution, but not the range, of the stimulus set. As we will see, the two groups performed quite similarly, suggesting that this small change had negligible effects. In each session, two measurements per condition were collected for the experimental trials (137-cm rod), yielding 30 experimental trials per testing session; this included 18 endpoint-walking trials and 12 length-walking trials. There were 15 and 18 additional nonexperimental trials in each session for the task cue and no task cue groups, respectively.

Stimuli and Procedure. The testing location, stimuli, and procedure were much the same as those in Experiment 1. As before, the participants walked without vision for approximately 3 min under the guidance of a sighted experimenter prior to testing and then donned hearing protectors to attenuate auditory localization cues. In the length-walking trials in Experiment 2, however, the participants paced out the rod's length, starting from the observation location; the walking direction on these trials was held constant $\left(45^{\circ}\right.$ to the right) for both depth and frontal rods. A tactile guide stood just behind the starting position to help properly orient the participants before they began walking. The guide consisted of a 1-m-long horizontal rod, mounted at a height of $1 \mathrm{~m}$. A drawing was used to emphasize that rod length, not rod distance, should be indicated on length-walking trials.

On each trial, the participants viewed the stimulus rod for $5 \mathrm{sec}$. The experimenter announced the response type ("length" or "near/ far/left/right endpoint") before the blindfold was lowered for one group and afterward for the other. The participants then executed the required response, using their own self-paced walking speed, and their stopping point was recorded. The participants were rotated to face the starting point and were guided back to the starting point without vision. No error feedback was given. The data were averaged across repetition within observers before analysis with an ANOVA.

\section{Results and Discussion}

Length-walking responses. Again, despite strong reasons to expect that walked indications of rod length would show evidence of foreshortening, there was none $[F(1,14)=1.90, p>.05]$. Furthermore, this lack of depth foreshortening was again coupled with large walking errors (see Figure 4A and Figure 2, Experiment 2). When walking to indicate the length of a $137-\mathrm{cm}$ rod, the participants walked an average of $245 \mathrm{~cm}$ - an overshoot of $79 \%$. Only 3 out of 384 responses undershot the required value, and some responses were well over $450 \mathrm{~cm}$ (Figure 3, Experiment 2). As in Experiment 1, rod length did not exert a strong effect on the amount of overwalking (Table 1). Both the lack of foreshortening and the large overshooting errors, then, are quite robust and clearly do not require that the walking be directed to the rod's location. Experiment 2 showed that these results are not critically dependent on there being several meters of nonvisual walking preceding the length-walking response, nor are they dependent on the multiple starts and stops required by two-segment paths.

The response certainty and monocular/binocular manipulations did not elicit any reliable effects or interactions. Thus, neither the opportunity to prepare for a particular response while viewing the stimulus nor binocular cues appear to play a crucial role in this context. The monocularbinocular differences in Experiment 1, then, were likely due to random sampling differences between the two groups. For clarity, Figure 4 collapses over this variable. There was a main effect of rod distance $[F(2,28)=11.03$, $p<.001]$. Pairwise planned comparisons $(p=.05)$ showed that responses for the 10 -m distance were somewhat larger than those for the other two distances, which in turn did not differ reliably from each other. This effect was absent in both the walking responses and the verbal estimates in Experiment 1, so its cause here is unclear. There were no other reliable main effects or interactions.

Endpoint walking. Figure 4B shows the results of the trials in which the observers attempted to walk to only the near or only the far endpoint of the depth rods. As in Experiment 1 , there was a main effect of endpoint $[F(1,14)=$ $15.65, p=.001]$ and an endpoint $\times$ target distance interaction $[F(2,28)=7.26, p=.003]$. Planned comparisons showed reliable endpoint effects at the 4- and 10-m target distances ( $p=.045$ and $<.001$, respectively) and a marginally reliable effect at $7 \mathrm{~m}(p=.07)$. There was a reliable response certainty group $\times$ endpoint $\times$ monocular/binocular viewing interaction $[F(1,14)=4.93, p=$ 

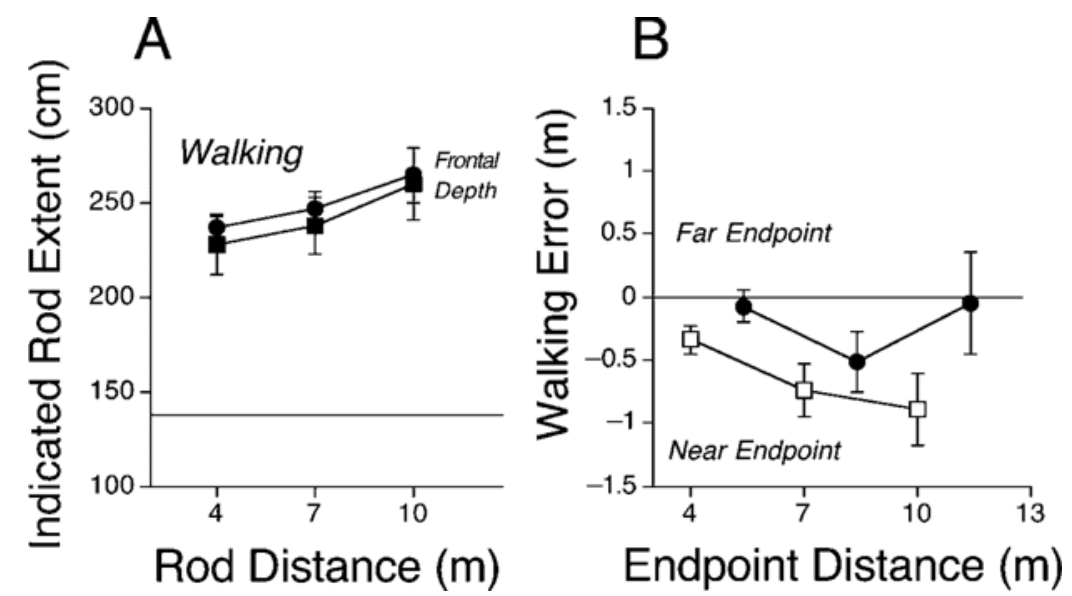

Figure 4. (A) Mean indicated rod extents for frontal and depth rod orientations in Experiment 2. The horizontal line indicates accurate responding. The overshooting errors are even larger than those in Experiment 1. All error bars denote plus or minus one standard error of the mean, and the data are averaged over monocular/binocular viewing and the response certainty manipulation. (B) Mean signed error in walking from the origin to the near and far endpoints of a $137-\mathrm{cm}$ rod oriented in depth. The horizontal line indicates accurate performance. The endpoints served as single targets on separate trials. There is again a clear difference in the pattern of errors, depending on the target endpoint. All error bars denote plus or minus one standard error of the mean, and the data are averaged over monocular/binocular viewing and the response certainty manipulation.

.043]. Planned comparisons showed that there were reliable endpoint effects for all combinations of response certainty and viewing conditions (all $p$ s $<.04$ ), except for the cued group under monocular viewing $(p=.65)$. The reasons for this interaction are unclear. There were no other main effects or interactions involving response certainty and monocular/binocular manipulations.

This group of observers tended to undershoot both the near and the far endpoints slightly. Although there was slightly more undershooting of both endpoints than in Experiment 1, a similar context effect emerged in which the participants responded to the near and the far endpoints differently; there is again a vertical separation of the two error curves in Figure 4B, with far endpoint error being on top. Notably, this context effect occurred even though the task was nominally egocentric in nature and did not involve walking to two endpoints in sequence. Apparently, the mere presence of the rod induced the participants to treat the endpoints differently.

Derived length responses. Given the observed pattern of errors in the endpoint-walking responses, one may wonder how responses directed to the two endpoints of each stimulus compare with the length-walking responses for that stimulus. Is the separation between the stopping locations for the two endpoints (obtained on separate trials) comparable to the direct indications of rod length in length-walking trials? One way to address this issue is to construct a derived measure of rod length by comparing stopping locations across individual endpoint-walking trials. These derived length responses are not strictly comparable with the direct responses, because the derived measure confounds within- and between-trials variability. Responses to frontally oriented rods also raise problems, because small variations at the origin in orienting toward the right-hand endpoint translate into large variations in the final stopping location. We therefore did not statistically compare the derived and the direct length responses. However, by focusing on responses to the $137-\mathrm{cm}$ rod oriented in depth, the mean signed error for the derived length responses was $+48.6 \mathrm{~cm}( \pm 9.49 \mathrm{~cm})$, as compared with $+104.58 \mathrm{~cm}( \pm 7.25 \mathrm{~cm})$ for the direct length responses. Thus, there was considerable inaccuracy even in the derived length responses, even though the task did not entail any explicit indication of rod length. This inaccuracy, however, may be somewhat reduced in comparison with direct length judgments.

\section{EXPERIMENT 3}

Experiment 2 replicated the findings in the present Experiment 1 and in Experiment 2 of Loomis et al. (1992) by showing a lack of depth foreshortening in the walking responses. However, the participants in the present Experiment 2 always turned $45^{\circ}$ to the right before initiating their walking responses, whereas those in the previous experiments walked in different directions once they had reached the stimulus location, depending on the orientation of the stimulus. Although Experiment 2 replicated the previous results, it may be that the replication was a fortuitous by-product of the particular response orientation used. To continue exploring the robustness of the response isotropy, in Experiment 3, we tested 
walking in three different directions (straight forward along the median sagittal plane and after $45^{\circ}$ and $90^{\circ}$ right turns). It also increased the size of the stimulus extents to include stimuli of 137,244 , and $351 \mathrm{~cm}$. Systematic manipulation of the stimulus extent permitted an assessment of whether any overwalking that was observed was proportional to the extent or tended to remain constant with increases in the stimulus extent.

\section{Method}

Participants. Fourteen adults ( 7 women and 7 men) were paid to participate in this experiment. The ages ranged from 20 to 24 years (median: 22 years). All were naive as to the purposes of the experiment.

Design and Stimuli. The experiment took place in a well-lit indoor gymnasium. On each trial, the observer binocularly viewed two vertical rods resting on the floor. No devices that attenuated or masked ambient sounds were used. The rods were white wooden dowels, $17.5 \mathrm{~cm}$ tall and $1.0 \mathrm{~cm}$ in diameter. Each rod was supported in the vertical position by two rigid wires extending inconspicuously to the floor behind the rod. One rod was positioned directly in front of the observer at an unchanging distance of $6.75 \mathrm{~m}$. The other was positioned so that it was aligned with the first rod either in depth (in the observer's median sagittal plane) or in a frontoparallel plane. There were three possible separations between the rods: 137,244 , and $351 \mathrm{~cm}$. On each trial, the observer was asked to orient his or her body in one of three possible directions before walking, to indicate the separation between the stimulus rods: (1) straight ahead, (2) a $45^{\circ}$ rightward turn, or (3) a $90^{\circ}$ rightward turn. The various pairings of rod separation, alignment, and response direction were fully crossed, and the presentation order was completely randomized. Three measurements were collected per condition, for a total of 54 trials.

Procedure. Before testing, the participants completed a single practice trial without error feedback, to familiarize them with the procedure. This trial involved walking forward without vision approximately $4 \mathrm{~m}$ without assistance and then walking back to the origin under the guidance of the experimenter. On each trial, the participant viewed the workspace and the two stimulus rods for several seconds and then covered his or her eyes with a blindfold. The experimenter announced the response direction to be used, and then the participant oriented his or her body in the specified direction and attempted to walk a distance that matched the separation of the stimulus rods. The instructions did not call attention to the viewing angle but did emphasize that the goal was to produce a walking response that equaled the physical separation between the two rods. When the participant stopped walking, an experimenter measured the distance traveled and then guided the participant back to the starting position without providing error feedback. In the interest of streamlining the procedure for the return trajectory, the participants did not turn in place to face the starting position before returning to the origin but, instead, walked backward under the guidance of the experimenter until the starting position was reached.

\section{Results and Discussion}

The signed error scores were averaged over repetition and analyzed with an ANOVA. This analysis showed that there was no main effect of stimulus orientation [depth vs. frontal; $F(1,13)=2.86, p=.115]$. This result is consistent with the walking results from Experiments 1 and 2, as well as with previous work (Loomis et al., 1992). More germane to the goals of Experiment 3, however, is the walking direction variable; this manipulation resulted in a nonsignificant trend $[F(2,26)=2.74, p=$
.083]. Although the main effect was not reliable, planned comparisons involving just the two response orientations used in the present Experiment 1 and in Loomis et al. (1992) showed that responses in the median sagittal plane were reliably larger than the $90^{\circ}$ responses $(p=.028)$. Responses made after a $45^{\circ}$ right turn did not differ significantly from either sagittal walking nor $90^{\circ}$ walking (both $p \mathrm{~s}>.20$ ). By averaging over stimulus orientation and stimulus extent, the mean signed errors were +30.2 , +24.1 , and $+16.2 \mathrm{~cm}$ for the sagittal, $45^{\circ}$, and $90^{\circ}$ walking conditions, respectively (see also Figure 2B, Experiment 3 , which shows mean responses only for the $137-\mathrm{cm}$ stimulus, for comparison with the other experiments). Thus, there is evidence that reproductions of exocentric intervals by nonvisual walking are indeed influenced by walking direction, apart from any effect due to stimulus orientation. ${ }^{1}$ More research is needed to determine the cause of this directional bias. One possibility is that, when one walks in the sagittal plane in exocentric tasks, there is a kind of representational inertia in self-motion sensing, so that a small initial amount of locomotion must occur before self-motion sensing begins to proceed normally.

The directional walking bias was observed when the participants indicated the magnitude of spatial extents near the observation location. The present experiments do not speak to whether or not a similar bias would be observed in exocentric walking responses conducted at the stimulus location. Nevertheless, it is important to note that exocentric walking responses conducted near the observation point were robustly isotropic, even when the directional walking bias was taken into account. The participants in Experiment 2 always walked in the same direction $\left(45^{\circ}\right)$ when responding, thereby nullifying any influence of a directional walking bias. Similarly, in Experiment 3, there was again little evidence of depth compression when the response direction was held constant (see Figure 2A, Experiment 3).

There was no main effect of stimulus extent on the signed errors $[F(2,26)=0.36, p=.70]$. However, there was a stimulus extent $\times$ stimulus orientation interaction $[F(2,26)=4.72, p=.018]$. Planned comparisons showed that this interaction was driven primarily by responses for the 351-cm stimulus ( $p \leq .05$ ); when the $351-\mathrm{cm}$ stimulus was oriented in depth versus frontally, the mean signed errors were +2.88 and $+34.11 \mathrm{~cm}$, respectively. Responses for depth versus frontal stimuli did not differ significantly for the $137-$ and $244-\mathrm{cm}$ stimulus extents (both $p \mathrm{~s}>.30$ ). The signed errors averaged +27.46 and $+24.62 \mathrm{~cm}$ for the $137-$ and $244-\mathrm{cm}$ extents, respectively. Thus, for the largest extent, there was some evidence of depth compression in the walking responses. There were no other reliable main effects or interactions in this analysis.

Interestingly, the overshooting errors in this study were considerably smaller than those in Experiments 1 and 2 (see Figure 2B and Table 1). One difference that might account for the smaller errors is that the partici- 
pants in the earlier experiments were exposed to a much greater total amount of nonvisual walking. In the first two experiments, the participants walked without vision for $3 \mathrm{~min}$ in a familiarization phase prior to testing, whereas the participants in Experiment 3 received no such familiarization. In Experiment 4, we investigated the possibility that the prolonged exposure to nonvisual walking prior to testing in Experiments 1 and 2 was responsible for the observed overshooting errors.

\section{EXPERIMENT 4}

Exposure to locomotion, coupled with slower-thanappropriate optic flow, is known to result in overshooting when individuals subsequently attempt to walk without vision to previewed targets (Rieser, Pick, Ashmead, \& Garing, 1995). Given that observers walk accurately before they experience this unusual coupling of locomotion and optic flow, and assuming that the observers intend to walk to the same location before and after such exposure, this result suggests that the locomotor control system has been recalibrated. That is, the same intended amount of locomotion now results in a different amount of actual locomotion than it did before exposure to the altered relationship between locomotion and vision. Other changes in locomotor calibration occur even after exposure to locomotion when vision is completely obscured (Anstis, 1995; Durgin, Fox, \& Kim, 2003; Durgin \& Pelah, 1999). ${ }^{2}$ When one walks without vision, there is a mismatch between self-motion signals specified by visual versus nonvisual sensory systems (e.g., proprioception, efference copy, or vestibular signals). The nonvisual signals indicate that the individual is moving, whereas the lack of optic flow caused by the occlusion of vision may effectively signal that the individual is stationary (Durgin et al., 2003). As individuals are exposed to nonvisual walking, the effective gain of the nonvisual self-motion signals may be attenuated in order to reduce the mismatch between the nonvisual and visual selfmotion signals (Barlow, 1990; Welch, 1986). In this view, after exposure to nonvisual walking, more physical walking is required to produce an amount of perceived self-motion that equals the intended amount of walking.

Consistent with this idea, Durgin, Pelah, and Amirud$\operatorname{din}(1998)$ found substantial overshooting in a nonvisual egocentric walking task after $90 \mathrm{sec}$ of blindfolded running $(8.3 \mathrm{kph})$ on a treadmill. Rieser et al. (1995, Experiment 2), meanwhile, found no evidence of aftereffects of exposure to $10 \mathrm{~min}$ of nonvisual walking ( $4 \mathrm{kph}$ ). There are several methodological differences between these two egocentric walking studies that might underlie the apparent discrepancy; we will return to this issue in the Experiment 4 Discussion section. Nevertheless, it is possible that exocentric and egocentric tasks draw upon somewhat different mechanisms, and given that the participants walked without vision for approximately $3 \mathrm{~min}$ prior to Experiments 1 and 2, the overshooting errors exhibited in those two exocentric tasks may well have been due to recalibration-like processes. A primary goal of Experiment 4 was to evaluate this possibility. We tested one group of participants who had no exposure to nonvisual walking just prior to testing (as was the case in Experiment 3) and another group who were exposed to 3 min of nonvisual walking prior to testing (as was the case in Experiments 1 and 2). We were interested not only in the relative amount of overshooting errors between the two groups, but also, as in Experiment 3, in the pattern of constant errors as the required response distance increased.

If exposure to nonvisual walking in a warm-up phase recalibrates subsequent nonvisual walking responses in exocentric tasks, presumably this calibration gradually will be restored to something like its pre-warm-up state as an individual is exposed to normal visually guided locomotion. A second goal of Experiment 4 was to begin an investigation of the dynamics of this process. A systematic study of this issue is beyond the scope of this article, but as a first step, we exposed one group of observers to nonvisual walking for $3 \mathrm{~min}$ and then tested them in an exocentric nonvisual walking task; we then exposed this group to an equal duration of walking with vision and retested them on the same task. A second group of participants walked with vision for 3 min prior to each of the two test phases. We compared the two groups' performances on the first test to evaluate the effect of exposure to nonvisual walking on walked indications of exocentric extents. We compared their performances on the second test to evaluate the effect of subsequent reexposure to walking with vision, over and above any effects that might accrue simply by virtue of participation in the same test a second time. We used a between-groups manipulation of the amount of exposure to nonvisual walking because we wanted to minimize the total amount of nonvisual walking available to one group as much as possible and yet still evaluate the effect of a second exposure to the test.

\section{Method}

Participants. Twenty-four adults (13 women and 11 men) participated in this experiment in exchange for course credit. The ages ranged from 18 to 30 years (median: 19 years). All were naive as to the purposes of the experiment.

Apparatus and Design. The stimulus rods, viewing conditions, and testing environment were the same as those in Experiment 3. In Experiment 4, however, the rods were oriented only in a frontoparallel plane. In each of two test phases, three rod separations $(137,244$, and $351 \mathrm{~cm}$ ) were presented three times apiece in random order. Each test phase was preceded by a warm-up phase, during which the observers walked for $3 \mathrm{~min}$. The observers were randomly assigned to participate in either the no-vision warm-up group or the vision warm-up group. The groups were distinguished by whether or not the participants walked with vision during the warm-up phase prior to Test 1 . All the participants walked with vision during the warm-up phase immediately prior to Test 2 . The male:female ratio was 5:7 in the no-vision warm-up group and 6:6 in the vision warm-up group.

Procedure. In the Test 1 warm-up, as in Experiment 3, the participants completed a single practice trial without error feedback to familiarize them with the procedure. Following this practice trial, 
the participants in the no-vision warm-up group walked for approximately $3 \mathrm{~min}$ without vision under the guidance of an experimenter. The participants in the vision group also walked for $3 \mathrm{~min}$, accompanied by an experimenter, but with eyes open. This warmup phase was conducted in a nearby location set apart from the testing area, and no sound-attenuating or sound-masking devices were worn. The walking speed was established by the experimenter, who attempted to walk at a "normal" pace - that is, neither hurried nor uncharacteristically slow. On the basis of the typical distance traversed in $3 \mathrm{~min}$, this speed was approximately $5 \mathrm{kph}$. At the termination of this warm-up phase, the participants were left standing at the starting position for Test 1.

In Test 1, all the participants wore sound-attenuating headphones throughout, and the procedure was identical for both groups. On each experimental trial, the participant raised a blindfold to view the workspace and the two stimulus rods. After several seconds, the participant donned a blindfold, oriented his or her body approximately $45^{\circ}$ to the right, and then attempted to walk without vision a distance that matched the physical separation of the stimulus rods. When the participant stopped walking, an experimenter measured the distance traveled and then guided the participant back to the starting position. All the participants walked backward during this return to the starting position. No error feedback was given.

In the Test 2 warm-up, all the participants walked for approximately 3 min with eyes open, accompanied by an experimenter. The walking speed was again established by the accompanying experimenter at his or her normal pace. The participants were instructed to pay attention to their surroundings as they walked. Test 2 was conducted in the same way as Test 1 .

\section{Results}

The signed error scores were averaged over repetition and analyzed with an ANOVA. There were clear group differences, particularly in the first test phase. The ANOVA revealed a marginal main effect of warm-up group $[F(1,22)=4.23, p=.052]$ and a reliable test phase $\times$ warm-up group interaction $[F(1,22)=5.07, p=.03]$. We predicted that the participants who had walked without vision prior to Test 1 would subsequently walk farther in
Test 1 than those who had walked with vision; pairwise two-tailed $t$ tests $(d f=22)$ between the two groups showed that this was true only for the largest stimulus extent: the resulting $p$ values were $.18, .08$, and .003 for the $137-$, $244-$, and $351-\mathrm{cm}$ extents, respectively. Unlike the results of Experiment 3, there was a reliable main effect of stimulus extent $[F(2,44)=32.33, p<.001]$, and this was in turn qualified by a reliable extent $X$ warm-up group interaction $[F(2,44)=6.15, p=.004]$ and by a three-way stimulus extent $\times$ warm-up group $\times$ test phase interaction $[F(2,44)=6.87, p=.002]$. As we will discuss shortly, these effects and interactions involving the extent variable were driven primarily by the responses of the no-vision warm-up group, whose errors were particularly influenced by the stimulus extent. There were no other reliable comparisons (see Figure 5).

The between-groups design leaves open the possibility that these results were due to chance differences between groups that existed prior to testing, but we think this unlikely given the size and systematic nature of the effects, particularly for the largest extent (see Figure 5, Test 1). In Experiment 3, constant errors did not vary with increases in stimulus extent. By striking contrast, however, the constant errors of the no-vision warm-up group in Test 1 increased markedly as stimulus extent increased; this was confirmed by pairwise planned comparisons (all $p \mathrm{~s}<.05$ ). We interpret this result as evidence that exposure to nonvisual walking during the warm-up session reduced the effective gain of the nonvisual self-motion signals, with the result that each pace was registered to be somewhat shorter than it actually was. In this view, as the stimulus extents increased, the participants needed to produce increasingly longer physical distances to create a perceived amount of self-motion that subjectively matched the previewed stimulus extents. By

\section{Warm-up} for Test 1

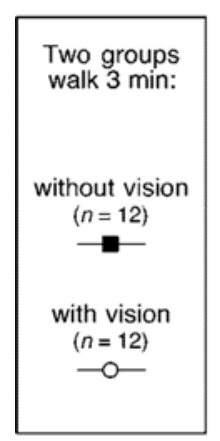

Test 1 (Nonvisual Walking)

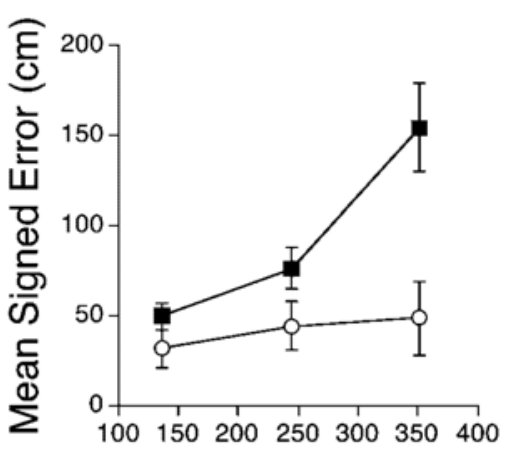

Stimulus Extent (cm)
Warm-up for Test 2
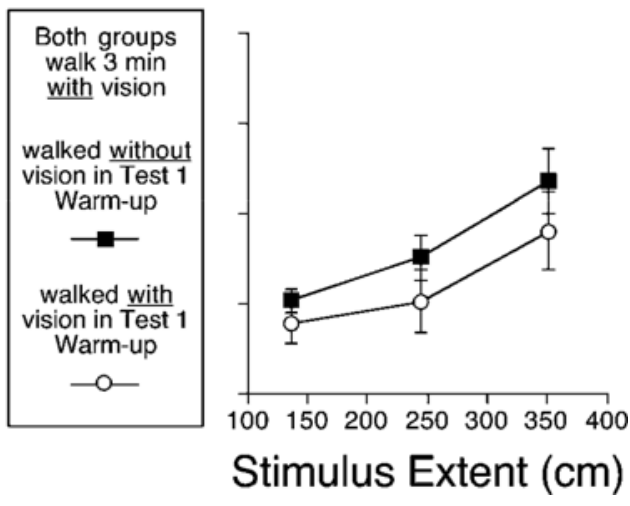

Figure 5. Mean signed walking error for two groups of participants in each of two test periods in Experiment 4. One group walked for 3 min without vision during the Test 1 warm-up period, and the other walked with vision during this period. Both groups walked with vision for $3 \mathrm{~min}$ prior to completing Test 2 . Tests 1 and 2 were identical and entailed walking without vision to indicate the magnitude of a previously viewed frontal extent. Responses for three stimulus extents are shown. Error bars denote plus or minus one standard error of the mean. 
contrast, the errors of the participants who walked with vision in the Test 1 warm-up were very similar across all stimulus extents ( $p>.05$ for all pairwise comparisons), a finding that accords well with the results of Experiment 3.

When the no-vision warm-up group was allowed to walk with vision for $3 \mathrm{~min}$ prior to Test 2 , their performance did not change dramatically from that shown in Test 1. Pairwise contrasts showed that the constant errors in Test 2 again depended strongly on stimulus extent, with all combinations of stimulus extents producing significantly different errors $(p<.05)$. In comparing this group's performance between Test 1 and Test 2, pairwise contrasts $(p=.05)$ showed that although responses for the 137- and 244-cm extents did not change significantly, constant errors for the largest extent reliably dropped in Test 2. Apparently, after the participants had been exposed to nonvisual walking for 5-10 min (including the warm-up and the first test phase), an additional 3 min of exposure to the normal coupling of vision and locomotion was not sufficient to cause substantial changes in walking performance for relatively short walking distances (within $3 \mathrm{~m}$ ). For longer distances, however, there was a tendency toward less overshooting after reexposure to visual control of locomotion.

The performance of the vision warm-up group in Test 2 also did not change dramatically from that in Test 1 . Pairwise contrasts of the constant errors for each stimulus extent ( $p=.05$ ) showed that the responses for the 137and 244-cm extents were unchanged in Test 2 , relative to Test 1 , whereas errors for the largest extent did differ significantly. Specifically, for this extent only, there was some tendency to overshoot during the second exposure to the testing phase. This could be evidence of recalibration, but considering the complex pattern of experience with visual and nonvisual walking available to this group in Test 2, such a conclusion should be treated as tentative until confirmed by future research.

\section{Discussion}

We interpret the group differences in Test 1 as evidence that exposure to walking without vision just prior to testing tends to increase the magnitude of responses when individuals use nonvisual walking to indicate previewed exocentric intervals. Considering just the $137-\mathrm{cm}$ stimulus extent, the effect is too small here to entirely account for the large overshooting errors for a comparable stimulus in Experiments 1 and 2. The participants in the earlier experiments, however, were exposed to much more nonvisual walking during testing, by virtue of repeatedly walking to and from targets up to $10 \mathrm{~m}$ away. The relation between the amount of exposure to nonvisual walking and the amount of overshooting in this task has yet to be worked out in detail, but it is reasonable to expect that this much additional exposure would be associated with larger overshooting errors than we observed in Experiment 4 . It thus seems quite likely that a significant portion of the large constant errors seen in the present Experiments 1 and 2 and in Loomis et al. (1992) was due to recalibration of the locomotor control system following prolonged exposure to nonvisual walking.

As was mentioned earlier, Durgin et al. (1998) found evidence of aftereffects of exposure to nonvisual running on a treadmill, whereas Rieser et al. (1995, Experiment 2) found no aftereffects of nonvisual walking. These studies differed in terms of speed of locomotion during adaptation, as well as duration of adaptation. In addition, both studies differed from Experiment 4 in other methodological details (e.g., characteristics of the adapting surface, egocentric vs. exocentric nature of the task, amount of familiarization with nonvisual walking before testing, etc.). The relative importance of these variables remains poorly understood. Future studies exploring these differences promise to shed light on the limiting conditions for the development of walking aftereffects.

Several previous studies involving nonvisual walking have provided participants with a warm-up session (without error feedback) prior to collecting data (e.g., Corlett \& Patla, 1987; Elliott, 1987; Loomis et al., 1992; Philbeck \& Loomis, 1997), often with the nominal goal of building up the participants' feelings of confidence and safety while walking without vision. Whether or not these subjective feelings play a role, Experiment 4 suggests that even several minutes of exposure to nonvisual walking is sufficient to cause a systematic increase in nonvisual walking responses. One implication of these findings is that if a testing session is fairly lengthy, these changes could occur during the session itself, thereby contributing to the within-subjects variable error. These findings also suggest that if conclusions are to be drawn about the calibration of normal visually guided walking on the basis of responses in nonvisual walking tasks, the results should be interpreted in light of the possibility that some recalibration might occur during testing.

The constant errors for the no-vision warm-up group may appear to depart from the results of the present Experiments 1 and 2 and of Experiment 2 in Loomis et al. (1992), in that the earlier experiments showed no apparent dependence on stimulus extent (Table 1). If this effect is real, it may be that after familiarization with nonvisual walking, the dependence of walking errors on stimulus extent is nonlinear and becomes more pronounced for longer extents.

Finally, several studies in which the aftereffects of exposure to sensory mismatches during locomotion have been investigated have addressed the issue of whether the aftereffects are the result of adaptation of peripheral sensory receptors or, instead, of recalibration at a more central level (Earhart et al., 2001; Pick, Rieser, Wagner, \& Garing, 1999; Rieser et al., 1995). Durgin et al. (2003) have argued for a more central origin of recalibration in the case of one-legged locomotion, on the basis of the finding that the amount of recalibration in a hopping task depended on the visual information available during adaptation, and not simply on the peripheral motor activity of the leg. Pick et al. have found evidence of both central and peripheral recalibration processes in the case 
of exposure to two-legged rotational locomotion. One result that might potentially support the idea of peripheral adaptation would be finding no aftereffects when there is an equal amount of locomotor activity in two opposite directions (Earhart et al., 2001; Pick et al., 1999). The vision warm-up group of Experiment 4 received very nearly the same amount of exposure to nonvisual forward walking as to backward walking. This group tended to overwalk slightly, but we did not test for aftereffects after prolonged backward walking. Thus, it remains possible that exposure to forward and backward nonvisual walking produces recalibration in both directions. More research is required to settle these issues.

\section{GENERAL DISCUSSION}

Previous work has shown that when the magnitudes of exocentric spatial intervals are indicated by nonvisual walking, the response pattern differs from that obtained by visual matching of spatial intervals (Loomis et al., 1992). Here, we found that similar differences exist when walking responses are compared with verbal reports; specifically, verbal responses are relatively well scaled but exhibit depth foreshortening, whereas walking responses are quite poorly scaled but do not exhibit foreshortening. The discrepancies remain when the stimulus configuration is equated across tasks, and more surprisingly, even when participants are prevented from directing their walking to the location of the stimulus itself. Reproducing the stimulus extent near the observation point further enhances the similarity between the motoric (walking) and the nonmotoric (verbal and visualmatching) tasks, and yet the walked indications of spatial extents in Experiment 2 again showed no evidence of depth foreshortening under these conditions. By eliminating stimulus configuration and response location discrepancies as primary factors underlying the motoric and nonmotoric response differences, Experiments 1 and 2 have considerably constrained the possible explanations by ruling out two especially likely candidates.

The pronounced overshooting in exocentric nonvisual walking tasks appears to be due largely to recalibration of locomotor control following prolonged exposure to nonvisual walking, although more research is needed to more precisely determine the extent to which this is true. Relatively simple recalibration processes cannot explain all of the puzzling aspects of nonvisual exocentric walking tasks, however. The fact remains that when observers walk without vision to indicate the location of a single previously viewed target location, their responses are typically quite accurate, whereas nonvisual walked indications of exocentric spatial intervals exhibit large overshooting errors. This holds true even when both response types are conducted after several minutes' exposure to nonvisual walking and the two response types are randomly interleaved (Experiment 2). Thus, walking responses in tasks that are nominally egocentric still yield a somewhat different pattern than do walking responses in nominally exocentric tasks. The explanation for these differences is currently unknown. Notably, Experiment 2 showed that even when participants direct their nonvisual walking to a single rod endpoint, their responses differ in another way from the usual pattern seen in egocentric walking tasks. The typical pattern is that constant errors do not depend strongly on target distance (e.g., Rieser et al., 1990); by contrast, Experiments 1 and 2 showed clear evidence of a context effect, in which constant errors varied systematically according to which rod endpoint served as the target. It is possible that the presence of the rest of the rod or, more generally, of a salient nearby stimulus affects the perceptual localization of an individual point on a well-structured ground plane (Meng \& Sedgwick, 2002; Sinai, Ooi, \& He, 1998). More research is needed to determine the source of this context effect and how it affects nonvisual egocentric and exocentric walking responses.

Does the lack of depth compression exhibited by walking responses reflect a corresponding isotropy in the underlying spatial representation? As we have seen, several theoretical frameworks have been proposed in which the perceptual, motor, or cognitive requirements of a task might enhance the accuracy of the representation underlying a particular behavioral response (e.g., Bingham \& Pagano, 1998; Bridgeman, 1999; Loomis et al., 1996; Milner \& Goodale, 1995; Post \& Welch, 1996; Vishton et al., 1999). It may be that the task requirements surrounding the verbal and visual-matching tasks engender a more depth-foreshortened spatial representation, whereas a more accurate representation is constructed when a walking response is required. The robustness of the isotropy in walking responses is certainly striking; given that the response isotropy is observed both when participants must walk for several seconds without vision to get to the stimulus location and when they respond much more immediately but displaced from the stimulus, the notion of there being a single isotropic representation underlying both cases is appealingly parsimonious. Meanwhile, in this conceptualization, the overshooting errors in the walking responses do not reflect poor scaling in the underlying spatial representation but, rather, miscalibration of the locomotor control system.

As compelling as this straightforward interpretation might be, the possibility that a depth-foreshortened spatial representation underlies the walking responses cannot be ruled out by the currently available data. Some space perception theorists have proposed that egocentric distances are increasingly underperceived beyond $3-4 \mathrm{~m}$, in keeping with the decreasing effectiveness of the primary egocentric distance cues (e.g., Foley, 1991; Gogel, 1979). The depth-foreshortened visual matches and verbal estimates of exocentric intervals are consistent with this view. Accurate indications of the target locations beyond 3-4 m, as well as indications of the separation between locations, can be maintained by processes that "correct" for perceptual errors (Foley, 1978, 1985; Gogel $\&$ Da Silva, 1987). Within this framework, one source of 
correction is thought to be based on cognitive processing, such as expectations about where the target "should" be (Carlson, 1977). Processes that determine the calibration of motor responses may have the effect of biasing behavioral responses toward accuracy with respect to physical target locations and, to this extent, could also be thought of as correcting for perceptual errors (Bingham, Zaal, Robin, \& Shull, 2000; Rieser et al., 1995). The large overshooting errors in the exocentric walking tasks of Experiments 1 and 2 indicate that if correction processes were influencing the responses, these processes were not entirely effective in minimizing error. It may be that the overshooting reflects a recalibration of the locomotor control system, perhaps at a relatively central stage, while a yet more cognitive process operates to correct for the depth foreshortening in the underlying spatial representation. If this is true, however, additional research is needed to explain why the cognitive processes that so robustly correct for the perceptual foreshortening when a walking response is required are not similarly engaged when visual matches or verbal reports are required.

The experiments presented here highlight the complexity of the linkage between perception and action. The large overwalking errors exhibited when observers use nonvisual walking to indicate exocentric intervals can be explained, to some extent, by a recalibration of locomotor control as a result of exposure to walking without vision. The lack of depth compression exhibited in nonvisual walking responses was found to be quite robust. This may reflect a corresponding isotropy in the spatial representation controlling the response, but more research is required to resolve the issue. This problem is particularly important in light of the abundance of evidence that perceived spatial layout is strongly depth-foreshortened under the conditions tested here (e.g., Beusmans, 1998; Loomis et al., 1992; Toye, 1986; Wagner, 1985). Despite its complexity as a response measure, the remarkable robustness of nonvisual walking in terms of its isotropy makes it a valuable tool for investigating the spatial representations that are constructed in natural well-structured environments.

\section{REFERENCES}

Abrams, R. A., \& Landgraf, J. Z. (1990). Differential use of distance and location information for spatial localization. Perception \& Psychophysics, 47, 349-359.

ANstis, S. (1995). Aftereffects from jogging. Experimental Brain Research, 103, 476-478.

BARLOW, H. B. (1990). A theory about the functional role and synaptic mechanism of visual after-effects. In C. Blakemore (Ed.), Vision: Coding and efficiency (pp. 363-375). New York: Cambridge University Press.

Berthoz, A., Amorim, M.-A., Glasauer, S., Grasso, R., Takei, Y., \& ViAUd-Delmon, I. (1999). Dissociation between distance and direction during locomotor navigation. In R. G. Golledge (Ed.), Wayfinding behavior: Cognitive mapping and other spatial processes (pp. 328348). Baltimore: Johns Hopkins University Press.

Beusmans, J. M. H. (1998). Optic flow and the metric of the visual ground plane. Vision Research, $\mathbf{3 8}, 1153-1170$.
Bingham, G. P. (1993). Perceiving the size of trees: Biological form and the horizon ratio. Perception \& Psychophysics, 54, 485-495.

Bingham, G. P., \& Pagano, C. C. (1998). The necessity of a perceptionaction approach to definite distance perception: Monocular distance perception to guide reaching. Journal of Experimental Psychology: Human Perception \& Performance, 24, 145-168.

Bingham, G. P., ZaAl, F., Robin, D., \& Shull, J. A. (2000). Distortions in definite distance and shape perception as measured by reaching without and with haptic feedback. Journal of Experimental Psychology: Human Perception \& Performance, 26, 1436-1460.

BRENNER, E., \& SMEETs, J. B. (1996). Size illusion influences how we lift but not how we grasp an object. Experimental Brain Research, 111, 473-476.

BRIDGEMAN, B. (1999). Separate representations of visual space for perception and visually guided behavior. In G. Aschersleben \& T. Bachmann (Eds.), Cognitive contributions to the perception of spatial and temporal events (Vol. 129, pp. 3-13). Amsterdam: Elsevier.

CARLSON, V. R. (1977). Instructions and perceptual constancy judgments. In W. Epstein (Ed.), Stability and constancy in visual perception: Mechanisms and processes (pp. 217-254). New York: Wiley.

Corlett, J. T., \& Patla, A. E. (1987). Some effects of upward, downward, and level visual scanning and locomotion on distance estimation accuracy. Journal of Human Movement Studies, 13, 85-95.

Durgin, F. H., Fox, L. F., \& KiM, D. H. (2003). Not letting the left leg know what the right leg is doing: Limb-specific locomotor adaptation to sensory-cue conflict. Psychological Science, 14, 567-572.

Durgin, F. H., \& Pelah, A. (1999). Visuomotor adaptation without vision? Experimental Brain Research, 27, 12-18.

Durgin, F. H., Pelah, A., \& Amiruddin, S. (1998). Measures of visuomotor adaptation to anomalous optic flow [Abstract]. Investigative Ophthalmology \& Visual Science, 39, S1094.

Earhart, G. M., Jones, G. M., Horak, F. B., Block, E. W., Weber, K. D., \& Fletcher, W. A. (2001). Forward versus backward walking: Transfer of podokinetic adaptation. Journal of Neurophysiology, $\underline{\mathbf{8 6}}$, 1666-1670.

ELLIOTT, D. (1987). The influence of walking speed and prior practice on locomotor distance estimation. Journal of Motor Behavior, $\underline{\mathbf{1 9}}_{2}$ 476-485.

Foley, J. M. (1978). Primary distance perception. In R. Held, H. W. Leibowitz, \& H.-L. Teuber (Eds.), Handbook of sensory physiology: Vol. VIII. Perception (pp. 181-213). Berlin: Springer-Verlag.

Foley, J. M. (1985). Binocular distance perception: Egocentric distance tasks. Journal of Experimental Psychology: Human Perception \& Performance, 11, 133-149.

FoLEY, J. M. (1991). Binocular space perception. In D. Regan (Ed.), Binocular vision (Vol. 9, pp. 75-92). Boca Raton, FL: CRC Press.

Franz, V. H., Fahle, M., Bülthoff, H. H., \& Gegenfurtner, K. R. (2001). Effects of visual illusions on grasping. Journal of Experimental Psychology: Human Perception \& Performance, 27, 1124-1144.

Gillam, B., \& Chambers, D. (1985). Size and position are incongruous: Measurements on the Müller-Lyer figure. Perception \& Psychophysics, 37, 549-556.

GoGEL, W. C. (1979). The common occurrence of errors of perceived distance. Perception \& Psychophysics, 25, 2-11.

Gogel, W. C., \& DA SiLVA, J. A. (1987). A two-process theory of the response to size and distance. Perception \& Psychophysics, 41, 220-238.

Goodale, M. A., Jakobson, L. S., \& KeIllor, J. M. (1994). Differences in the visual control of pantomimed and natural grasping movements. Neuropsychologia, 32, 1159-1178.

HafFenden, A. M., \& GoOdALE, M. A. (1998). The effect of pictorial illusion on prehension and perception. Journal of Cognitive Neuroscience, 10, 122-136.

LoOmis, J. M., DA Silva, J. A., FuJita, N., \& Fukusima, S. S. (1992). Visual space perception and visually directed action. Journal of Experimental Psychology: Human Perception \& Performance, 18, 906-921.

Loomis, J. M., Da Silva, J. A., Philbeck, J. W., \& FukUsima, S. S. (1996). Visual perception of location and distance. Current Directions in Psychological Science, 5, 72-77.

Mack, A., Heuer, F., Villardi, K., \& Chambers, D. (1985). The dissociation of position and extent in Müller-Lyer figures. Perception \& Psychophysics, 37, 335-344. 
Marotta, J. J., Behrmann, M., \& Goodale, M. A. (1997). The removal of binocular cues disrupts the calibration of grasping in patients with visual form agnosia. Experimental Brain Research, 116, 113-121.

MARTENIUK, R. G., \& Roy, E. A. (1972). The codability of kinesthetic location and distance information. Acta Psychologica, 36, 471-479.

Meng, J. C., \& Sedgwick, H. A. (2002). Distance perception across spatial discontinuities. Perception \& Psychophysics, 64, 1-14.

Milner, A. D., \& Goodale, M. A. (1995). The visual brain in action. Oxford: Oxford University Press.

Norman, J. F., Todd, J. T., Perotti, V. J., \& Tittle, J. S. (1996). The visual perception of three-dimensional length. Journal of Experimental Psychology: Human Perception \& Performance, 22, 173-186.

Pagano, C. C., \& Bingham, G. P. (1998). Comparing measures of monocular distance perception: Verbal and reaching errors are not correlated. Journal of Experimental Psychology: Human Perception \& Performance, 24, 1037-1051.

PHILBECK, J. W. (2000). Visually directed walking to briefly glimpsed targets is not biased toward fixation location. Perception, 29, 259272.

Philbeck, J. W., \& Loomis, J. M. (1997). Comparison of two indicators of perceived egocentric distance under full-cue and reduced-cue conditions. Journal of Experimental Psychology: Human Perception \& Performance, 23, 72-85.

PiCK, H. L., JR., RieSER, J. J., WAgner, D., \& Garing, A. E. (1999). The recalibration of rotational locomotion. Journal of Experimental Psychology: Human Perception \& Performance, 25, 1179-1188.

Post, R. B., \& Welch, R. B. (1996). Is there dissociation of perceptual and motor responses to figural illusions? Perception, 25, 569-581.

Proffitt, D. R., Creem, S. H., \& Zosh, W. D. (2001). Seeing mountains in mole hills: Geographical-slant perceptions. Psychological Science, 12, 418-423.

Rieser, J. J., Ashmead, D. H., Talor, C. R., \& Youngquist, G. A. (1990). Visual perception and the guidance of locomotion without vision to previously seen targets. Perception, 19, 675-689.

Rieser, J. J., Pick, H. L., JR., AshmeAd, D. H., \& Garing, A. E. (1995). Calibration of human locomotion and models of perceptual-motor organization. Journal of Experimental Psychology: Human Perception \& Performance, 21, 480-497.

Rock, I. (1986). The description and analysis of object and event perception. In K. R. Boff, L. Kaufman, \& J. P. Thomas (Eds.), Handbook of perception and human performance (Vol. 2, pp. 33.31-33.71). New York: Wiley.

SinAI, M. J., OoI, T. L., \& He, Z. J. (1998). Terrain influences the accurate judgement of distance. Nature, 395, 497-500.

Soechting, J. F., \& Flanders, M. (1992). Moving in three-dimensional space: Frames of references, vectors, and coordinate systems. Annual Review of Neuroscience, 15, 167-191.

THOMSON, J. A. (1983). Is continuous visual monitoring necessary in visually guided locomotion? Journal of Experimental Psychology: Human Perception \& Performance, 9, 427-443.
ToYe, R. C. (1986). The effect of viewing position on the perceived layout of space. Perception \& Psychophysics, 40, 85-92.

VindRas, P., \& ViVIani, P. (1998). Frames of reference and control parameters in visuomanual pointing. Journal of Experimental Psychology: Human Perception \& Performance, 24, 569-591.

Vishton, P. M., Rea, J. G., CutTing, J. E., \& NuñEZ, L. N. (1999). Comparing effects of the horizontal-vertical illusion on grip scaling and judgment: Relative versus absolute, not perception versus action. Journal of Experimental Psychology: Human Perception \& Performance, 25, 1659-1672.

WAGNER, $\bar{M}$. (1985). The metric of visual space. Perception \& Psychophysics, 38, 483-495.

WELCH, R. B. (1986). Adaptation of space perception. In K. R. Boff, L. Kaufman, \& J. P. Thomas (Eds.), Handbook of perception and human performance: Vol. 1. Sensory processes and perception (pp. 24.21-24.45). New York: Wiley.

Wraga, M., Creem, S. H., \& Proffitt, D. R. (2000). Perception-action dissociations of a walkable Müller-Lyer configuration. Psychological Science, 11, 239-243.

\section{NOTES}

1. An alternative way of grouping the data that illustrates the same point is according to the congruency of the stimulus orientation and the walking direction. Congruent response conditions would entail depth stimuli indicated by forward walking and frontal stimuli indicated by walking after a $90^{\circ}$ turn, $45^{\circ}$ incongruent conditions would entail walking at $45^{\circ}$ to indicate both depth and frontal stimuli, and $90^{\circ}$ incongruent conditions would entail depth stimuli indicated by walking after a $90^{\circ}$ turn and frontal stimuli indicated by forward walking. Averaging across stimulus extent, the mean response ratios (depth stimuli/width stimuli) generated by grouping the data in this way were $1.07,0.95$, and 0.91 for the congruent, $45^{\circ}$ incongruent, and $90^{\circ}$ incongruent responses, respectively; the associated standard errors were $0.04,0.02$, and 0.04 . This pattern of decreasing response ratios with decreasing stimulus/response congruence is a consequence of the tendency to walk farther in the straight-ahead direction. The response ratios (depth stimuli/frontal stimuli) in the $90^{\circ}$ incongruent condition, for example, are less than 1 because responses to frontal stimuli are generally larger than responses to depth stimuli.

2. Nonvisual manual reaches toward fixed previewed targets also tend to gradually increase in egocentric distance across trials (Bingham, Zaal, Robin, \& Shull, 2000; Vindras \& Viviani, 1998). Unlike the case of whole-body locomotion, however, there is no drift when the movements are repeatedly made entirely without vision (i.e., haptically defined target locations). Apparently, somewhat different processes are involved in the two cases, but more research is required to characterize these differences.

(Manuscript received January 15, 2002; revision accepted for publication August 14, 2003.) 